\title{
Perspective
}

\section{Integrated Care Model Developed by the Rwanda Biomedical Center for Decentralization of Psychological Interventions during Commemoration of Genocide against Tutsi in Rwanda}

\author{
Jean D. Iyamuremye ${ }^{1 *}$, Rebecca L. White ${ }^{2}$ \\ ${ }^{1}$ Mental Health Division, Rwanda Biomedical Center, Kigali, Rwanda, \\ ${ }^{2}$ University of Illinois at Chicago, Human Resource for Health, Kigali, Rwanda
}

\begin{abstract}
Global and national burden of mental disorders has been increasingly recognized in terms of responding to the need for explicit, cost-effective, evidence-based yet culturally sensitive interventions (WHO, 2013). In 2004 the first official commemoration of genocide against Tutsi in Rwanda, which included mental health interventions, was held in Kigali. At that time the mental health support was reactive, centralized, and overwhelmed by the need for their services. In 2013 the Rwandan Biomedical Center (RBC), of the Ministry of Health, created a decentralized network of well-trained mental health responders throughout Rwanda. The objective of this decentralized network was to provide most of the care in the community and avoid using higher levels of care. This article reviews the infrastructure and evaluates the new decentralized community-based system that is now utilized throughout Rwanda during commemoration of Genocide against Tutsi in Rwanda.
\end{abstract}

Key words: Integrated Care Model, psychological Interventions, genocide, Rwanda

\section{Introduction}

In 1994, Rwanda experienced genocide against Tutsi which led to an extensive loss of human life, talents, and resources. Consequently, the Rwandan society was severely impaired and could not function optimally. In addition, the genocide survivors were devastated by the loss of all they had known resulting in massive unspeakable suffering (Ministry of Health, 2012).

Each year from $7^{\text {th }}$ April to $13^{\text {th }}$ of July, for a period of 100 days, Rwanda commemorates the genocide against Tutsi. During this commemoration period, a large number of people suffer from mental health related issues and emotional crises (Dyregrov, Gupta, Gjestad, \& Mukanoheli, 2000). When such crises are not managed properly or when there is a lack of interventions, they may create extreme anxiety and panic among genocide survivors participating in the commemorative events. To provide care to them, the Rwandan Biomedical Center (RBC), Mental Health Division, organizes and coordinates mental healthcare during this time of the year all over the country.

\section{Historical Perspective - an Impetus for Change}

Each year, a large number of people have "emotional crises" during the commemoration period of the Genocide (Ministry of Health, 2015). The crises are often collective and create distress among the people who are participating in the events. Often accompanied by the crises, are extreme emotional episodes, which, when witnessed, create a resurgence of memories from the genocide itself. As a result, there arises re-traumatization that require intervention by mental health professionals, and hospitalization when severe.

In 2004 the first official commemoration, which included mental health interventions, was organized in Kigali. Since then, the mental health support was reactive, centralized, and annual, overwhelmed by magnitude of the required service. In response to this need, in 2013 the RBC created a decentralized network of well-trained mental health responders throughout Rwanda. They implemented a chain of communication and a plan for mass interventions to be available throughout the commemoration at each level of the health system in Rwanda.

Training of the workforce to assist the population during commemoration was a vast and organized project by

*Corresponding author: iyadamas@gmail.com 
the RBC. The workforce capacity building included specialized training in trauma and crisis interventions and early recognition and referral of patients suffering from mental illness. Trained members of the commemoration workforce included the Community Health Workers for each village (i.e. Umudugudu), which totaled 15,000. Health professionals trained included 500 nurses working in community health centers. Volunteers from community support organizations were trained including the Red Cross, the association for students who are survivors of the genocide (AERG), ambulance service providers known as SAMU, association of genocide survivors (IBUKA), and association of the widows of genocide (AVEGA). Following the training of the members association of survivors of genocide, it was found that there existed a strong correlation with self-care techniques and increased support for the community from well-respected individuals in their respective roles.

\section{Sensitization and Awareness of Trauma Inter- ventions}

In order to increase awareness of trauma and trauma reactions and to prepare the community for commemoration, the RBC mental health division used many modalities. These modalities included radio spots and pamphlets, which encouraged mental wellness within the Rwandan population, and highlighting prevention of trauma. The major contribution initiated in 2014 in the intervention model was the introduction of a 24-hour crisis hotline available for three months. Anyone can call 119 on all cell phone providers' networks and receive a live trained intervention worker with special skills to support and provide referral to that caller.

An annual press conference was held in 2014 where 30 journalists were briefed prior to the commemoration. $\mathrm{RBC}$ requested the journalists' assistance to inform the Rwandan population about healthy proactive behaviors and common issues that may arise during this time, and where the population can find help and assistance. During the community work, known as "Umuganda" in Kinyarwanda, in March 2014, the community leaders distributed important messages on how each community member needs to prepare the community commemora- tion sites and how to behave during this time. The RBC mental health division distributed concise guidelines for this to each of the 15,000 village leaders.

\section{Training Activities Instituted by Mental Health Division, Rwandan Biomedical Center}

Each year numerous training sessions to empower the workforce begin in February and continue until the end of March. For example in 2014, 45 mental health nurses from all district and referral hospitals were trained in crisis and trauma care interventions. The association for student survivors of genocide (AERG: French acronym) had a frontline workforce of 750 trained facilitators in the same year. These AERG students were initially trained in first aid skills and mental health issues, to assist with early recognition of those suffering in the commemoration ceremonies throughout Rwanda. The students then returned to train identified community members in supporting the people in mental health needs. The Red Cross volunteers numbering 150 were also part of the frontline during commemoration in 2014. In addition, Community Health workers numbering 15,000 received a two days training on crisis intervention and returned to their respective villages as a primary support person within each village throughout Rwanda. Likewise, 70 general nurses from Kigali City were also included in the training and these nurses played a major role in the support of patients in Kigali and surrounding areas. Finally, $80 \mathrm{ambu}-$ lance care providers known as SAMU received training to increase their interventional skill levels. The ambulance services workforces were important as they connected all three levels of psychological interventions utilized during the genocide commemoration.

\section{Organization of interventions}

The multidisciplinary teams were established and trained and have demonstrated their effectiveness. The protocols and systems of communication have been developed and utilized. The use of the transportation systems and the support of volunteers have been implemented successfully. Crisis Intervention was provided for all Rwandans during commemoration at three distinct levels as described in Figure 1. 


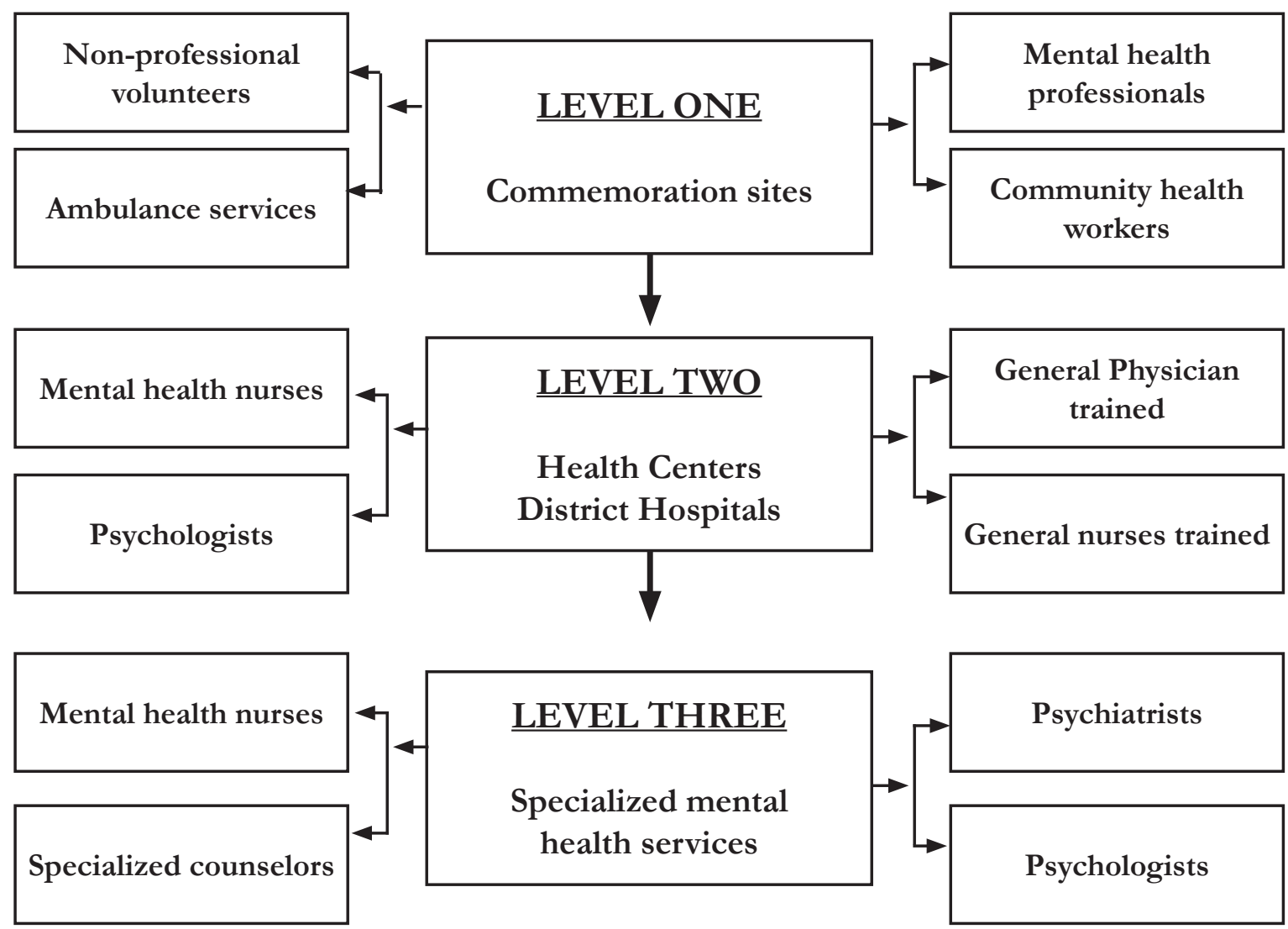

Figure 1. Intervention during commemoration of Genocide Care Delivery Organizational Chart

Level One: Interventions at Commemoration Sites

At the first level of care the AERG and Red Cross workers were on the front lines at all commemoration sites. They assisted the patients experiencing trauma reactions and help them to go to designated treatment rooms, which are staffed by mental health professionals, including mental health nurses, psychologists, and physicians. The patient is evaluated and if deemed to require a higher level of care, the patient is then transferred to the nearest Health Center at the District level by ambulance (SAMU).

\section{Level Two: Interventions at the General Health Facilities}

The level two of care intervention includes 500 health centers and district hospitals staffed by general nurses and physicians trained in trauma and crisis intervention techniques. The RBC mental health division prior to the commemoration trained these nurses. The Ministry of Health $(\mathrm{MOH})$ ensures these sites have availability of beds and blankets to ensure comfort for the patients. Emergency kits are provided for the nurses, which include medications for sedation in cases of acute agitation and anxiety responses. Ambulance services are coordinated to ensure timely transfer to a higher level of care if needed. The next step for a patient in acute crisis includes transfer to the District Hospital when needed within the level two of the intervention model. Specially trained mental health nurses and psychologists are at all 43 district hospitals and are the next phase of care for those patients suffering acutely from trauma and anxiety during the commemoration period.

Level Three: Interventions at Specialized Mental Health Service Sites

Complicated cases are transferred from the district hospitals via ambulance to the Kigali Teaching Hospital (CHUK) and are admitted to the Mental Health Department, and some cases are transferred to Ndera Neuropsychiatric Hospital (HNP-Ndera). Commonly, those transferred to the third level of care have been found to have comorbidities of preexisting mental health and/or substance abuse issues (Ministry of Youth, 2012; Ministry of Health, 2012).

\section{Results achieved}

Statistics from 2012-2014 in Kigali City, as an example, demonstrated the effectiveness of the innovations, including the networking, training and the effectiveness of the psychological intervention model which resulted in the reduction of patients seen at central level during the main week of commemoration at the 4 sites in Kigali 
from $7^{\text {th }}-13^{\text {th }}$ April from 2012 to 2014 .

The number of patients seen during the commemoration reduced from 613 patients in 2012 to 161 patients in 2013 and then went up to 377 patients in 2014. This is due to the fact that in 2014 commemoration was the $20^{\text {th }}$ anniversary of Genocide and more people participated in the event.

The 2014 commemoration was the $20^{\text {th }}$ anniversary of the genocide, many people participated and this required sufficient preparation in term of personnel and materials (Ministry of Health, 2015). In 2014 the decentralization of services was in full force and the community sites outside of Kigali city reported a total of 2096 patients. Of the 2096 patients treated at the community level, 706 were transferred to the health center level and 292 were transferred at treated at the district level.

In 2014 out of 377 patients received care in Kigali City, the majority of people $232(61.5 \%)$ received healthcare on commemoration sites, without any need for transfer to a health facility. One hundred and thirty five cases representing $135(35 \%)$ received interventions from the Community Health Centers in their respective sites. Only seven cases representing $7(1.8 \%)$ were referred to District Hospitals $(\mathrm{DH})$ and only three patients representing $3(0.7 \%)$ were referred from the District Hospitals to specialized mental health services (See Figure 2). This further supports the effectiveness of the model $(\mathrm{MOH}$, 2014).

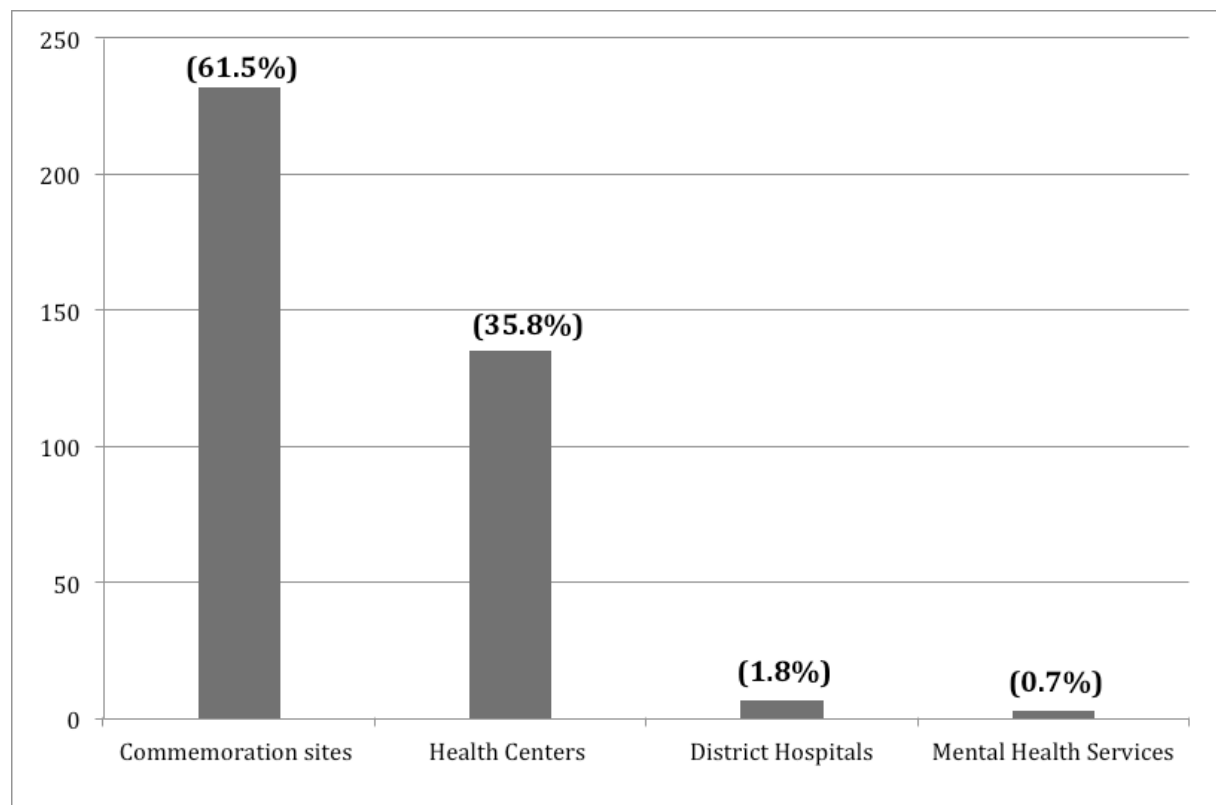

Figure 2. Number of people who received care by level of health facility/site in 2014

As shown in Figure 2, the majority of people (61.5\%) received healthcare on commemoration sites, without any need for transfer to a health facility. 135 cases representing 35\% received intervention from the Community Health Centers in their local communities. 7 cases representing $1.8 \%$ were then referred to District Hospitals $(\mathrm{DH})$ and only 3 patients representing $0.7 \%$ were referred from the District Hospitals to specialized mental health services (MOH,2015).

\section{Conclusion}

In conclusion, it has shown that the decentralization of commemoration activities and crisis interventions at the community level has decreased the rate of admissions in specialized mental health services which comprised the third level of care by a substantial percent.

As the majority of care was delivered at a community, this model is believed to be cost effective in term of use of resources, timeliness of care, financial savings, etc.

This psychological intervention model has been developed and implemented throughout Rwanda. The workforce consists of trained providers and the communication lines with standardized protocols are now utilized annually so that Rwandans can participate in commemoration safely and with the psychological support, if needed. The support is available within the local communities in which they live and allows all Rwandans to grieve with loved ones and the crisis interventions if needed can then 
be provided immediately. This decentralized care model allows easier accessibility with a strong professionally networked national workforce that is effective and efficient.

\section{Outlook for the Future}

The decentralization of the mental health care system and its use during the Commemoration of the Genocide has been a significant improvement implemented by the Ministry. Now the Rwandan people can be united and feel free to participate in the remembrance of their loved ones, allowing them to grieve fully in safe environment without damaging effects of re-traumatization and crisis. When we can remember in safe environment, we can fulfill our commitment to never forget as we move Rwanda forward to continued stability and healing. Continuous evaluation and research on the effectiveness of these interventions are needed in the future.

\section{Potential implications}

This innovative model of psychological support can serve as an example for other countries that have suffered from the effects of war and conflict. This integrated care program demonstrates that with the organized mobilization of a comprehensive multitier workforce, from volunteers to the highest level of trained individuals, a network can be created which utilizes the training and talents of all in concert. When this type of organization and training is implemented each member can contribute to the highest and best utilization of their talents and education in a way that creates the greatest service to all people. As there is too little data to say the model is generalizable, further study that includes a higher number of people and more detailed outcome measures is recommended for generalizability of the model to other settings.

\section{References}

Dyregrov, A., Gupta, L., Gjestad, R., \& Mukanoheli, E. (2000). Trauma exposure and psychological reactions to genocide among Rwandan children. Journal of Traumatic Stress, 13(1): 3-21.

Ministry of Health. (2012), National mental health policy in Rwanda, Kigali, $\mathrm{MOH}, \quad$ Kigali, Rwanda

Ministry of Health (2015). 20TH COMMEMORATION OF THE GENOCIDE AGAINST THE TUTSI: Report on bealth care during the commemoration period 7 to 13 April 2014. $\mathrm{MOH}$, Kigali, Rwanda

Ministry of Youth (2012). Prevalence of psychoactive substance use among youth in Rwanda, Kigali, Rwanda

Staub, E., Pearlman, L. A., Gubin, A., \& Hagengimana, A. (2005). Healing, reconciliation, forgiving and the prevention of violence after genocide or mass killing: An intervention and its experimental evaluation in Rwanda. Journal of Social and Clinical Psycbology, 24(3): 297-334.

WHO (2013). Mental Health action plan 2013-2020. Geneva 